\title{
A clinical and radiological objective tumor response with somatostatin analogs (SSA) in well-differentiated neuroendocrine metastatic tumor of the ileum: a case report
}

This article was published in the following Dove Press journal:

OncoTargets and Therapy

31 March 2015

Number of times this article has been viewed

\section{Chiara De Divitiis' \\ Claudia von $\mathrm{Arx}^{2}$ \\ Roberto Carbone 3 \\ Fabiana Tatangelo ${ }^{4}$ \\ Elena Di Girolamo 5 \\ Giovanni Maria Romano' \\ Alessandro Ottaiano' \\ Elisabetta de Lutio di \\ Castelguidone $^{3}$ \\ Rosario Vincenzo laffaioli' \\ Salvatore Tafuto' \\ On behalf of the European \\ Neuroendocrine Tumor \\ Society (ENETS) Center of \\ Excellence Multidisciplinary \\ Group for Neuroendocrine \\ Tumors in Naples (Italy) \\ 'Department of Abdominal \\ Oncology, National Cancer Institute \\ "Fondazione G. Pascale", Naples, Italy; \\ 2Department of Clinical Medicine \\ and Surgery, "Federico II" University, \\ Naples, Italy; ${ }^{3}$ Department of \\ Radiology, ${ }^{4}$ Department of Pathology, \\ ${ }^{5}$ Department of Endoscopy, National \\ Cancer Institute "Fondazione G \\ Pascale", Naples, Italy}

Correspondence: Salvatore Tafuto Department of Abdominal Oncology, Division of Medical Oncology, Istituto Nazionale Tumori "Fondazione G Pascale", via M. Semmola 80I3I, Naples, Italy

Email s.tafuto@istitutotumori.na.it

\begin{abstract}
Somatostatin analogs (SSAs) are typically used to treat the symptoms caused by neuroendocrine tumors (NETs), but they are not used as the primary treatment to induce tumor shrinkage. We report a case of a 63-year-old woman with a symptomatic metastatic NET of the ileum. Complete symptomatic response was achieved after 1 month of treatment with SSAs. In addition, there was an objective response in the liver, with the disappearance of secondary lesions noted on computed tomography scan after 3 months of octreotide treatment. Our experience suggests that SSAs could be useful for downstaging and/or downsizing well-differentiated NETs, and they could allow surgery to be performed. Such presurgery therapy could be a promising tool in the management of patients with initially inoperable NETs.
\end{abstract}

Keywords: neuroendocrine tumor, somatostatin analogs, octreotide, metastatic tumor of the ileum, radiological tumor response

\section{Introduction}

Neuroendocrine tumors (NETs) originating from the small bowel comprise the largest subgroup of NET within the gastroenteropancreatic system after pancreatic NET. They reflect the typical distribution pattern of the diffuse endocrine digestive system. Small intestinal NETs are second to appendiceal carcinoids in terms of prevalence, but they often prevail in clinical series, as they represent the most common (90\%) cause of carcinoid syndrome. The reported annual incidence rate according to the most recent literature is about 0.32 per 100,000 inhabitants in England, 0.33 per 100,000 inhabitants in Japan, 0.67 per 100,000 inhabitants in the United States, 0.81 per 100,000 inhabitants in Norway, and 1.12 per 100,000 inhabitants in Sweden..$^{1-6}$ The incidence does not show a sex preference, with a slight male preponderance in some series. The mean age at initial diagnosis is in the late 50 s, with the majority of cases occurring in the seventh decade. ${ }^{7}$ Furthermore, patients with small-bowel carcinoid tumors tend to have a positive family history for several cancers. Significant increases in the risk of small-bowel carcinoid tumor development were observed in relation to the presence of a family history of prostate cancer or colorectal cancer among patients' first-degree relatives. The risk of small-bowel carcinoid tumors was elevated among those whose siblings were affected by tongue or mouth cancer. ${ }^{7-10}$

The prognosis of small-bowel NET depends on both staging and grading, which are reflected in the World Health Organization (WHO) classification of 2010. Many authors reported 5-year tumor-specific survival rates of $100 \%$ for stage I and II, $97 \%$ for stage 3 , and $85 \%$ for stage 4 jejunoileal NET. Grading-dependent 5 -year tumor-specific survival rates were $94 \%$ for $\mathrm{G} 1,83 \%$ for $\mathrm{G} 2$, and $50 \%$ for the very rare $\mathrm{G} 3$ histotype. ${ }^{8-10}$ 
However, prognosis for small intestinal NET is considerably better than that for other small intestinal neoplasms such as lymphomas, adenocarcinomas, and sarcomas. ${ }^{1-13}$ Although recent data suggest better overall survival, the datasets are not completely comparable as they are analyzed at different tumor stages. However, as suggested by Yao et al the improvement of the overall outcome probably depends on new diagnostic tools, effective treatment options, and multidisciplinary sequential or simultaneous treatments.

The treatment of functional metastatic well-/intermediatedifferentiated NETs is based on octreotide (OCT) or lanreotide, which are analogs of somatostatins (SSAs). International guidelines have also indicated interferon alpha (IFN- $\alpha$ ) as an alternative treatment in refractory patients. ${ }^{14}$ However, no statistically significant effects on overall survival were shown in three randomized clinical trials that have investigated SSAs alone or in combination with IFN- $\alpha$. In one multicenter study of 68 patients evaluating subcutaneous OCT alone or in combination with IFN- $\alpha$ at 3 million units, five times per week, the 5 -year survival rate was prolonged in the combination group (5-years survival rate: $36.6 \%$ vs $56.8 \%$, respectively), but not in a statistically significant fashion $(P=0.13) .{ }^{15}$ Another three-arm trial compared subcutaneous lanreotide to IFN- $\alpha$ ( 5 million units, three times per week) alone or in combination. The objective response rates were rare $(\leq 7 \%)$ in all three arms, and tumor progression rates were nearly identical. ${ }^{16} \mathrm{~A}$ third randomized study of 109 patients compared subcutaneous OCT alone or in combination with IFN- $\alpha$ at 4.5 million units, three times per week. Survival in the combined arm was prolonged (35 months in single agent arm versus 51 months in combined arm), but it did not achieve statistical significance $(P=0.38)$. Response rates in both arms were $<6 \% .{ }^{17}$

Up until 2009, the principal role of SSAs in NETs was only in palliative and symptomatic care. The inhibition of the process of secretion is obtained by inhibiting the activity of adenil cyclase and calcium channels with the lowest intracytoplasmic levels of cyclic adenosine monophosphate. Modlin et $\mathrm{al}^{18}$ in a review article, observed symptomatic relief in $74.2 \%(61.9 \%-92.8 \%)$ and $67.5 \%(40.0 \%-100 \%)$ of gastroenteropancreatic NET patients, and biochemical response in 51.4\% (31.5\%-100\%) and 39.0\% (17.9\%-58\%) of these patients following treatment with Sandostatin LAR and Somatuline SR/Autogel, respectively.

In biological models, many authors demonstrated the direct and indirect antiproliferative effects of SSAs. An antiproliferative effect by SSAs could also be mediated by the SSA receptors (SSTRs). In particular, the apoptotic effect of SSAs is mediated through SSTR2 and SSTR3, and antiangiogenic activity has been reportedly mediated through SSTR2. ${ }^{19}$

The indirect effects of OCT develop through the inhibition of growth factors and trophic hormones, as well as via the inhibition of angiogenesis and the modulation of the immune response. Instead, the direct effects are expressed through the activation of phosphotyrosine phosphatase or mitogen-activated protein kinase that regulate cell proliferation, which is achieved through the specific proapoptotic action of upregulated endonucleases.

Only since 2009 have these aspects taken form in vivo; following the publication by the PROMID (Placebo-controlled prospective Randomized study on the anti-proliferative efficacy of OCT LAR in patients with metastatic neuroendocrine MIDgut tumors) study group, ${ }^{20}$ we have results about the use of OCT in a prospective, double-blind, Phase III trial where 85 metastatic midgut NET patients were randomized to receive either OCT long-acting release (LAR) $30 \mathrm{mg}$ every 28 days or placebo (PBO). This study demonstrated that the use of long-acting OCT more than doubled the time to progression (TTP) when compared with the PBO group (14.3 months versus 6 months, respectively), and that after 6 months of treatment, stable disease was observed in $66.7 \%$ of patients in the OCT LAR group and in $37.2 \%$ of patients in the PBO group. Functionally active and inactive tumors responded similarly as well.

With regard to the effects of OCT on liver metastasis in midgut NET patients, a post hoc analysis of the PROMID trial, submitted by Arnold to ASCO in $2013,{ }^{21}$ confirmed that the median TTP in the subgroup of patients with low $(\leq 10 \%)$ hepatic tumor load was 27.1 months, and it was 7.2 months (hazard ratio: 0.26 ; 95\% confidence interval [CI]: 0.14-0.49; $P<0.0001)$ in the OCT and PBO groups, respectively. In addition, the best responses were based on the stabilization of progressing disease or tumor shrinkage, although the latter occurred rarely. ${ }^{21}$ These important conclusions, confirming the antiproliferative and cytostatic effects of OCT in metastatic midgut NET patients, changed the National Comprehensive Cancer Network guidelines and, since 2011, OCT was indicated in metastatic, well-differentiated functional and nonfunctional NETs.

\section{A case report}

A 63-year-old female patient was admitted in May 2012 for recurrent abdominal pain (Numeric Analog Scale =6-7), weight loss ( $7 \mathrm{~kg}$ over 5 months), diarrhea (4-5 stools per day), and alternating subocclusive symptoms, anorexia, asthenia, and facial and neck flush (4 times per day) lasting for 6 months; 
she also had an Eastern Cooperative Oncology Group (ECOG) Performance Status (ECOG PS) of 2. Physical examination revealed diffuse abdominal sensitivity at palpation and hepatomegaly.

An abdominal computed tomography (CT) scan, performed in May 2012, showed multiple nodular liver lesions, of which the largest were in the fourth segment (S) (about $37 \times 35 \mathrm{~mm}$ ), which were extended in part to the fifth $\mathrm{S}$ and into the right lobe (seventh $\mathrm{S}$ and eighth $\mathrm{S}$ of about $23 \times 16 \mathrm{~mm}$ and $13 \mathrm{~mm}$, respectively). The hypervascular pattern of secondary lesions was compatible with a NET; an oval area of $3.2 \mathrm{~cm}$ (maximum diameter) with sharp margins compressing the ileal loops and with an expansive aspect was found in the anterior hypogastrium. An abdominal magnetic resonance imaging (MRI) scan confirmed the presence of liver metastases.

In July 2012, a liver biopsy was performed. Histological examination revealed the presence of neoplastic proliferation in the solid growth patterns of insular cells; immunohistochemistry (pan-cytokeratin-positive, chromogranin-positive, Synaptophysin-positive, CD56-negative/positive, and Ki-67 <20\%) was consistent with the diagnosis of NET (Figure 1).
Urinary excretion of 5-hydroxyindolacetic acid could not be determined for technical reasons. Chromogranin was high (131.6 U/L; range: $2-18 \mathrm{U} / \mathrm{L})$ in the absence of proton pump inhibitors or kidney failure.

An octreoscan, performed in September 2012, showed two areas of hyperaccumulation of the radiopharmaceutical receptor in correspondence with the hepatic parenchyma at the level of the fourth $\mathrm{S}$ and fifth $\mathrm{S}$, and of the mesogastric region; MRI revealed two nodular areas consistent with neoplastic tissue.

In October 2012, the case was discussed by the tumor board of our institution. Given the presence of symptomatic disease, the risk of occlusion, the presence of liver metastases alone, and in accordance with the European Neuroendocrine Tumor Society (ENETS) Consensus Guidelines, the patient underwent surgery.

A stenosing tumor was found in the terminal ileum. Lymph nodes that had increased in size were identified along the right colonic artery, ileum, and cecum. An ileotransversal terminolateral anastomosis was performed. Histopathologic examination revealed a malignant intestinal NET with lymph
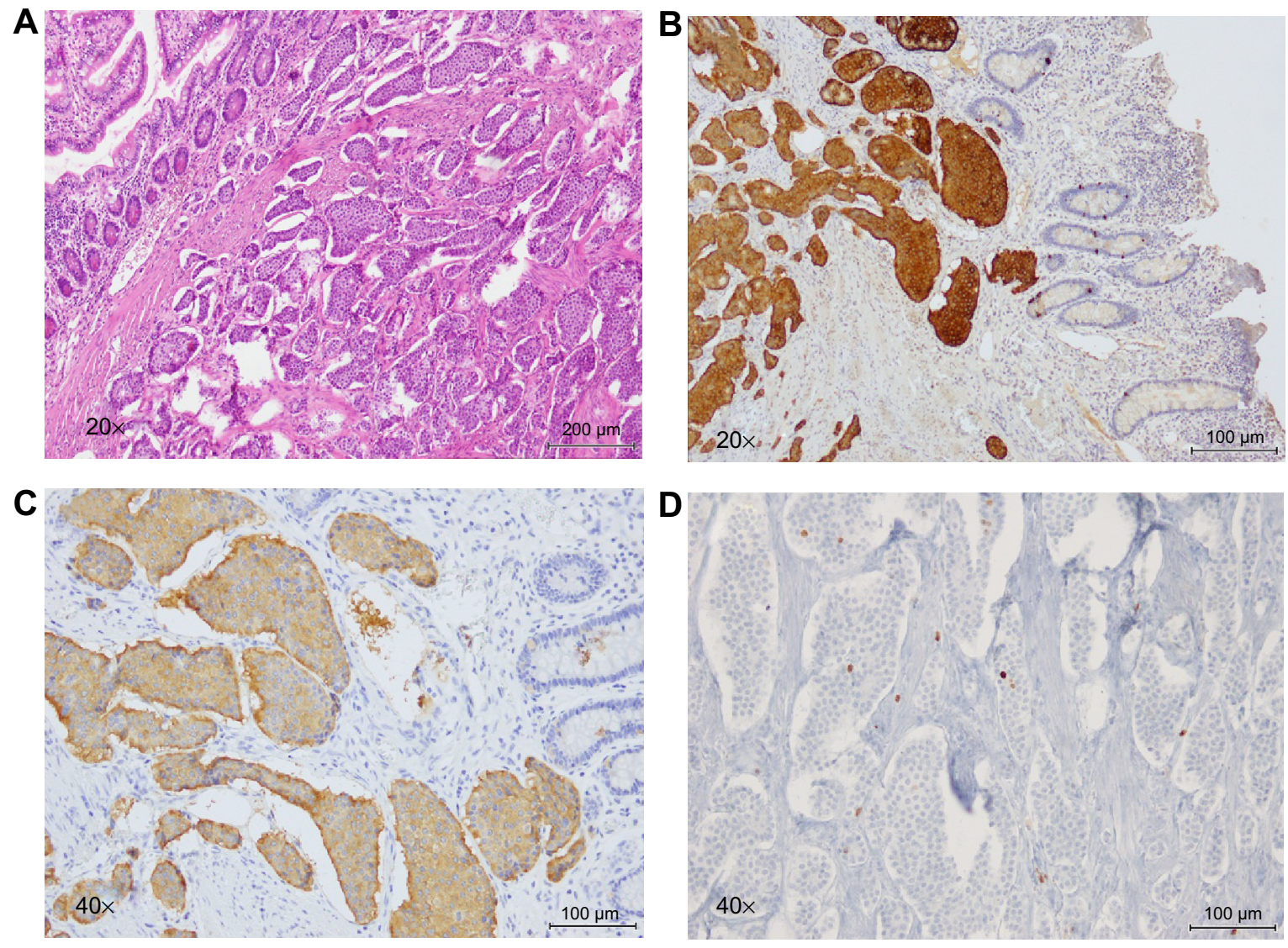

Figure I Histological examination with immunohistochemistry and hematoxylin-eosin staining (e/e).

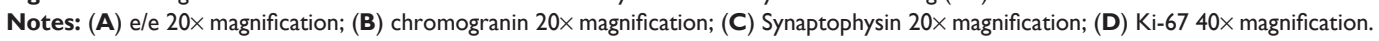


Table I After 3 months of OCT LAR $30 \mathrm{mg}$, an almost complete clinical response was observed in the patient, with symptom regression and PS recovery from ECOG PS 2 to ECOG PS 0

\begin{tabular}{lll}
\hline Symptomatic response & Basal & After 3 months \\
\hline ECOG PS & 2 & 0 \\
Diarrhea (stools per day) & $4-5$ & 0 \\
Abdominal pain (VNRS) & $6-7$ & $0-1$ \\
$\begin{array}{l}\text { Flushing (times per day) } \\
\text { Biochemical response }\end{array}$ & 4 & 0 \\
Chromogranin A level (U/L) & 131.6 & 12.9 \\
\hline
\end{tabular}

Abbreviations: VNRS, Verbal Numerical Rating Scale; OCT LAR, octreotide long-acting release; PS, Performance Status; ECOG, Eastern Cooperative Oncology Group.

node metastases: NET G1;22 and pT3 pN1 pv1 pR0 G2 (AJCC 2010). Postsurgical recovery was favorable, and the patient started therapy with OCT LAR $30 \mathrm{mg}$ intramuscularly every 28 days. At 1-month follow-up, the patient had marked improvement in her clinical conditions, with a significant weight increase. After 3 months of medical treatment, a complete clinical response with regression of symptoms, normalization of chromogranin levels, and PS recovery from ECOG PS 2 to ECOG PS 0 were obtained (Table 1). A total body CT scan showed a single secondary nodular lesion in the liver of about $34 \times 30 \mathrm{~mm}$ (versus $37 \times 34 \mathrm{~mm}$ ) (Figures $2 \mathrm{~A}$ versus 2B), with a slight decrease in its density. The other secondary lesions (present at the previous exam) were not clearly identified (Figures 3A versus 3B; Figures 3C versus 3D).
Thereafter, we submitted the case to the attention of our tumor board. We decided to re-evaluate the patient with a new total body CT scan after 2 months in order to confirm the clinical response in the liver to determine between performing hepatic surgery or a locoregional treatment (ie, transarterial embolization).

\section{Discussion}

This case report shows that OCT-based therapy is able to produce an objective response and clinical benefit in NET, whereas cytotoxic chemotherapy is rarely effective. SSAs are typically used to treat the symptoms caused by NET, but they are not used as a primary treatment to induce tumor shrinkage. In fact, in 70\%-90\% of patients, SSAs (OCT, lanreotide) are successful in the treatment of carcinoid syndrome. The antitumor effect of SSA appears to be weak, with an objective response rate of $10 \%$, even if used at high doses. However, disease stabilization rates up to $50 \%-60 \%$ have been reported. ${ }^{22,23}$ The PROMID trial showed that OCT LAR significantly lengthens the time to tumor progression when compared with PBO in patients with functionally active and inactive metastatic midgut NET. Eighty-five patients were randomized to receive Sandostatin LAR $30 \mathrm{mg}$ (number $[\mathrm{n}]=42$ ) or PBO ( $\mathrm{n}=43)$. After 6 months of treatment, patients receiving the drug had a $66 \%$ reduction in the risk of disease progression compared with patients taking PBO $(P=0.000072)$. Patients
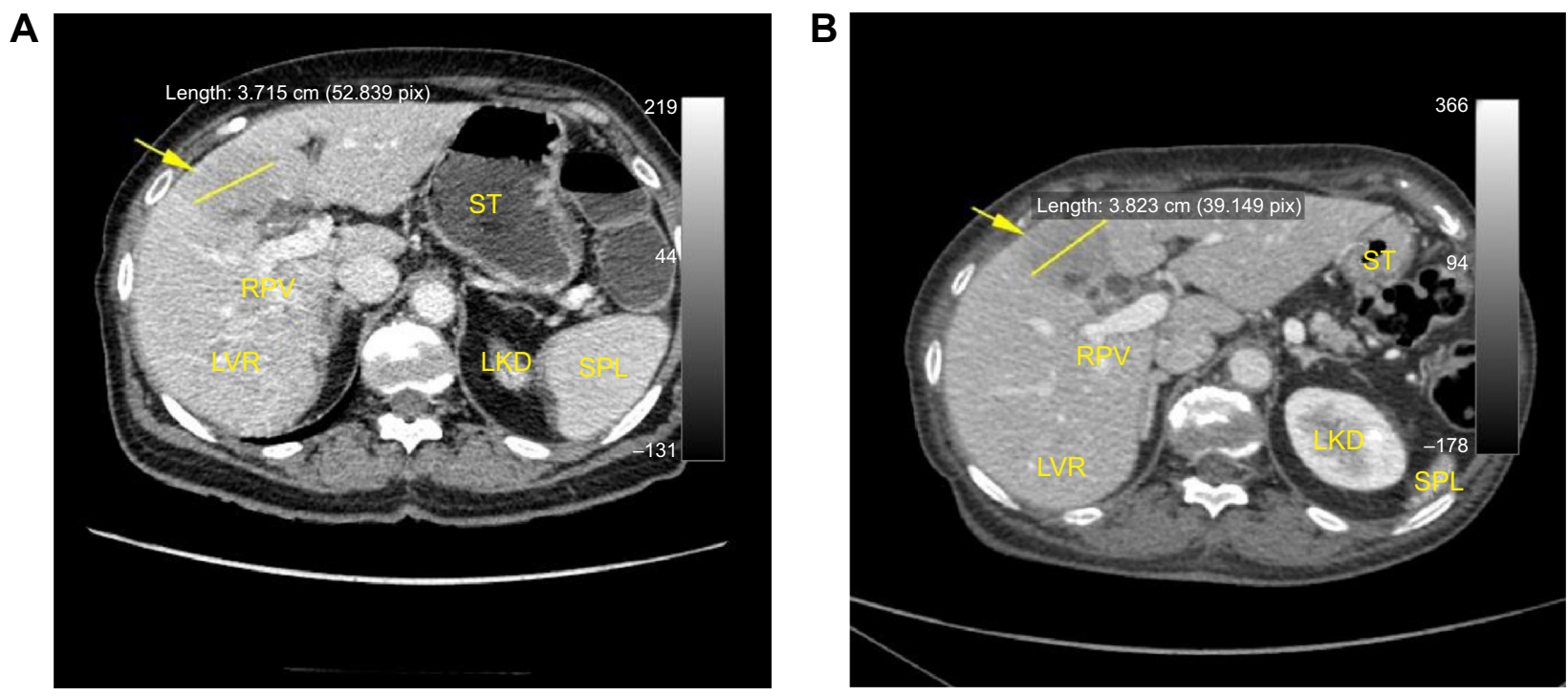

Figure 2 Liver metastatic NET.

Notes: (A and B) Contrast-enhanced CT. (A) A double-contrast study of the stomach; the difference between the abdominal parenchyma visualized in the two examinations lies in the different degrees of filling of the stomach and in the patient's different breaths. Before treatment, the hepatic lesion of the fourth segment shows inhomogeneous enhancement (yellow arrow). (B) After treatment, the lesion's size and density remain stable (yellow arrow). This is SD according to both the RECIST and CHOI criteria. Abbreviations: LVR, liver; RPV, right branch of the portal vein; ST, stomach; LKD, left kidney; SPL, spleen; NET, neuroendocrine tumor; CT, computed tomography; $\mathrm{SD}$, stable disease. 
A

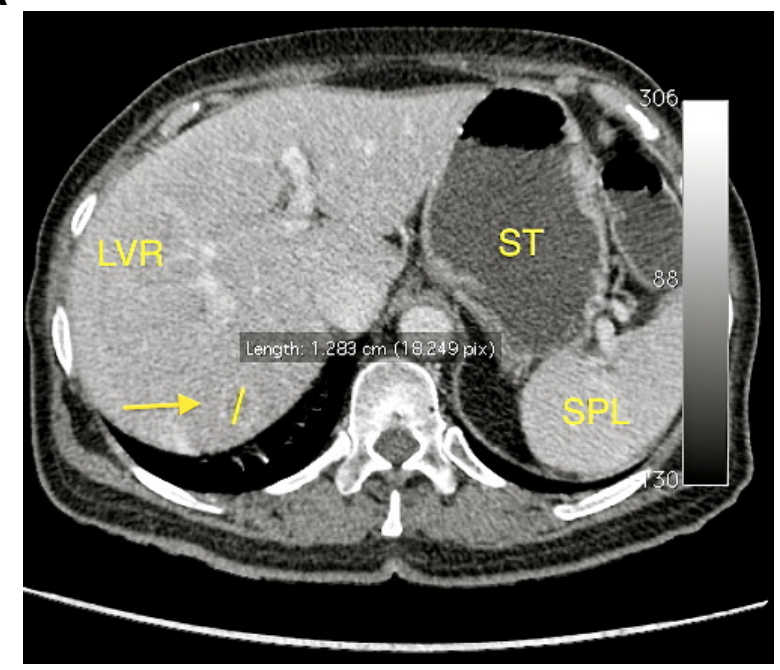

C

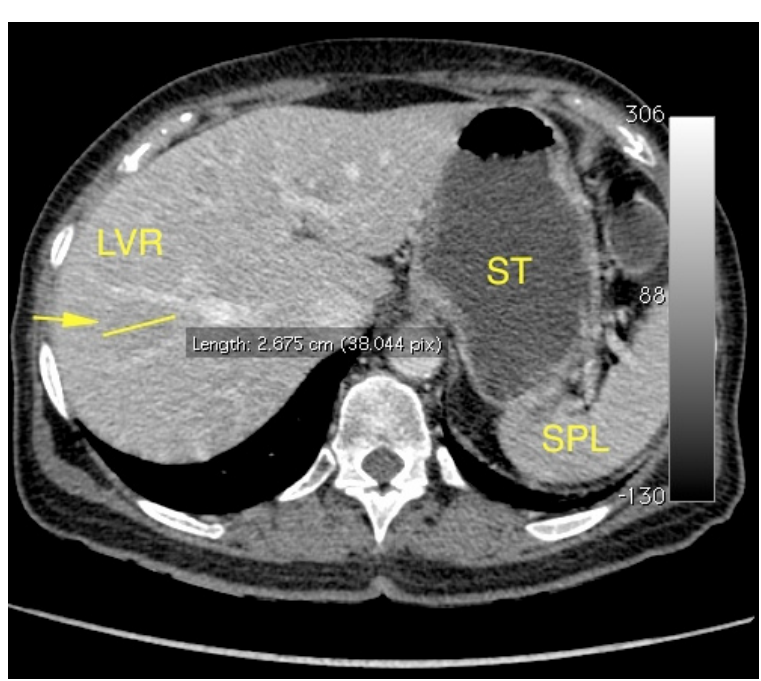

B

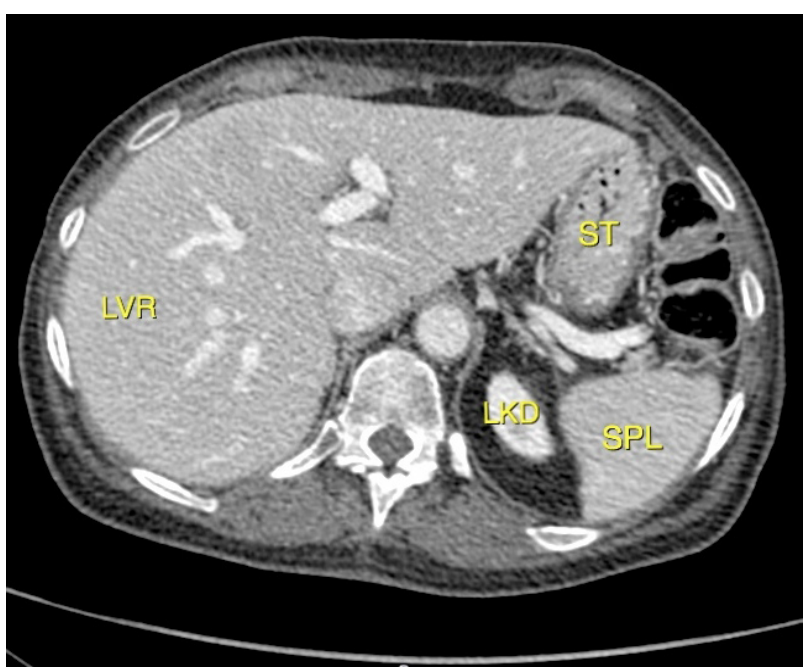

D

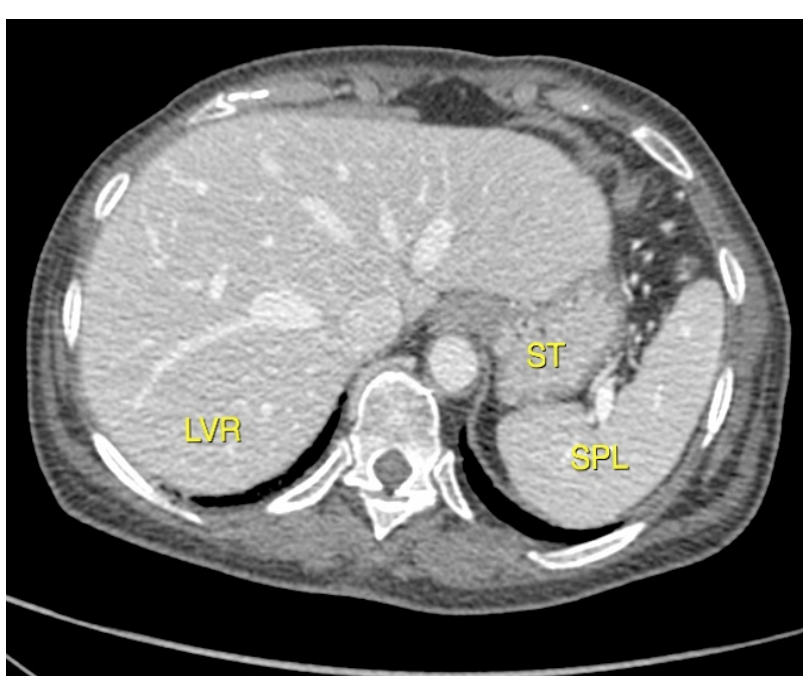

Figure 3 Liver metastatic NET.

Notes: (A-D) Contrast-enhanced CT. (A and $\mathbf{C}$ ) Double-contrast study of the stomach. The difference between the abdominal parenchyma visualized in the two examinations lies in the different degree of filling of the stomach and in the patient's different breaths. Before treatment, the hepatic lesions of the seventh and eighth segments appear as soft hypodense inhomogeneous lesions (yellow arrow). (B and D) After treatment, the lesions are not more appreciable.

Abbreviations: LVR, liver; ST, stomach; SPL, spleen; LKD, left kidney; NET, neuroendocrine tumor; CT, computed tomography.

treated with OCT LAR had no tumor progression for a median of 14.3 months, as compared with 6 months for patients taking the PBO. This beneficial effect was seen in either functioning (hormone secreting) or nonfunctioning (nonsecreting) NET. The results suggested that OCT LAR $30 \mathrm{mg}$ may have a direct antiproliferative effect in patients affected by NET. ${ }^{23}$

In this case report, symptom control was clearly obtained, resulting in an improvement in the patient's ECOG PS (0 versus 2) (ECOG) (Table 1). At diagnosis, the patient complained of recurrent abdominal pain, weight loss (7 kg over 5 months), anorexia, asthenia, and facial and neck flush lasting for 6 months. Also, the patient reported at least three emergency hospital admissions for abdominal pain and subocclusive symptoms. Postsurgery, after just 1 month of OCT treatment, the patient, once again in the presence of smaller liver metastasis, showed a complete regression of symptoms.

In addition, it is paramount to underline that an objective response in the liver, with the disappearance of secondary lesions during CT scan, was observed. The exceptional nature of the case is that the aforementioned results were obtained after just 3 months of treatment. These clinical, 
objective responses are commonly observed in metastatic colorectal cancer in patients treated with chemotherapy. The shrinkage obtained can lead the patient to liver resection or locoregional treatment, with a strong impact on survival time. In midgut metastatic NETs, this opportunity is rarely found using the SSAs alone and, of course, when it occurs, it can be due only to the proven antiproliferative activity of SSAs in NET.

In fact, the use of SSAs, especially OCT LAR, is recommended for only a few years with antiproliferative purposes in functioning and nonfunctioning midgut tumors. Moreover, SSAs are the recommended first-line therapy in nonfunctioning, progressive, small intestinal G1 NET. The treatment may be considered in therapy-naïve, metastatic patients without a prior observation period of spontaneous tumor growth. Also, it remains unclear if there is an individual benefit with respect to improved prognosis and increased survival, especially if treatment is started early after the initial diagnosis, as compared to a "watch-and-wait" strategy until tumor progression occurs. $^{22}$

In a recent randomized, double-blind, PBO-controlled, multinational study, some authors have demonstrated that lanreotide was associated with significantly prolonged progression-free survival among patients with metastatic enteropancreatic NETs of grade 1 or $2(\mathrm{Ki}-67<10 \%) .{ }^{24}$ The estimated rates of progression-free survival at 24 months were $65.1 \%(95 \%$ CI: 54.0-74.1) in the lanreotide group and $33.0 \%$ (95\% CI: 23.0-43.3) in the PBO group. ${ }^{24}$

However, there are no evidence-based data with respect to survival that support the early use of SSA. Thus, the extent of the disease and the tumor growth rate (in the case of prior observations of tumor growth) should be taken into account for the choice of the appropriate therapy. Thus, as in this case report, high tumor burden and/or high tumor growth rates may require early additional or alternative therapies. SSAs are usually used as a palliative treatment. The recommended dosage of OCT LAR for antiproliferative purposes is $30 \mathrm{mg}$ intramuscularly per month, as in the PROMID trial. However, in many cases, the intent of NET therapy has been changed from palliative to curative with the improvements in surgical techniques and in combined strategies. Moreover, the very low toxicity profile of OCT allows for many combinations with other therapeutic procedures and/or agents.

There are no reports dealing with the use of therapy with SSA as a presurgery strategy. However, our case suggests that SSAs could be useful for downstaging and/or downsizing the well-differentiated NETs, and they could allow surgery to be performed. Such a presurgery therapy could be a promising tool in the management of patients with initially inoperable NET. Future studies in selected patients are needed to address this issue.

This case also shows that a multidisciplinary approach improves the choice of the best and most appropriate treatment strategy. In fact, the presence of liver metastases largely influences the prognosis in all types of NET, and surgical resection with curative intent remains the gold standard in the treatment of liver metastases, achieving a survival rate of $60 \%-80 \%$ at 5 years with low mortality $(0 \%-5 \%)$ and acceptable morbidity (close to $30 \%$ ).

The use of SSA and surgery, as well as future targeted drugs, may increase the long-term control of some patients with advanced NET. An early multidisciplinary approach remains fundamental for the selection of the most appropriate treatments to adopt.

\section{Disclosure}

The authors report no conflicts of interest in this work.

\section{References}

1. Ellis L, Shale MJ, Coleman MP. Carcinoid tumors of the gastrointestinal tract: trends in incidence in England since 1971. Am J Gastroenterol. 2010;105(12):2563-2569.

2. Ito T, Sasano H, Tanaka M, et al. Epidemiological study of gastroenteropancreatic neuroendocrine tumors in Japan. J Gastroenterol. 2010; 45(2):234-243.

3. Yao JC, Hassan M, Phan A, et al. One hundred years after "carcinoid": epidemiology of and prognostic factors for neuroendocrine tumors in 35,825 cases in the United States. J Clin Oncol. 2008;26(18):3063-3072.

4. Hauso O, Gustafsson BI, Kidd M, et al. Neuroendocrine tumor epidemiology: contrasting Norway and North America. Cancer. 2008; 113(10):2655-2664.

5. Landerholm K, Falkmer S, Järhult J. Epidemiology of small bowel carcinoids in a defined population. World J Surg. 2010;34(7):1500-1505.

6. Niederle MB, Hackl M, Kaserer K, Niederle B. Gastroenteropancreatic neuroendocrine tumours: the current incidence and staging based on the WHO and European Neuroendocrine Tumour Society classification: an analysis based on prospectively collected parameters. Endocr Relat Cancer. 2010;17(4):909-918.

7. Ahlman H, Nilsson O, McNicol AM, et al; Frascati Consensus Conference participants. Poorly-differentiated endocrine carcinomas of midgut and hindgut origin. Neuroendocrinology. 2008;87(1):40-46.

8. Jann H, Roll S, Couvelard A, et al. Neuroendocrine tumors of midgut and hindgut origin: tumor-node-metastasis classification determines clinical outcome. Cancer. 2011;117(15):3332-3341.

9. Klimstra DS, Arnold R, Capella C, et al. WHO Classification of Tumours of the Digestive System. Lyon France: IARC; 2010.

10. Pape UF, Berndt U, Müller-Nordhorn J, et al. Prognostic factors of long-term outcome in gastroenteropancreatic neuroendocrine tumours. Endocr Relat Cancer. 2008;15(4):1083-1097.

11. Garcia-Carbonero R, Capdevila J, Crespo-Herrero G, et al. Incidence, patterns of care and prognostic factors for outcome of gastroenteropancreatic neuroendocrine tumors (GEP-NETs): results from the National Cancer Registry of Spain (RGETNE). Ann Oncol. 2010;21(9): 1794-1803.

12. Bergestuen DS, Aabakken L, Holm K, Vatn M, Thiis-Evensen E. Small intestinal neuroendocrine tumors: prognostic factors and survival. Scand J Gastroenterol. 2009;44(9):1084-1091. 
13. Strosberg J, Gardner N, Kvols L. Survival and prognostic factor analysis of 146 metastatic neuroendocrine tumors of the mid-gut. Neuroendocrinology. 2009;89(4):471-476.

14. Boudreaux JP, Klimstra DS, Hassan MM, et al; North American Neuroendocrine Tumor Society (NANETS). The NANETS consensus guideline for the diagnosis and management of neuroendocrine tumors: well-differentiated neuroendocrine tumors of the Jejunum, Ileum, Appendix, and Cecum. Pancreas. 2010;39(6):753-766.

15. Kölby L, Persson G, Franzén S, Ahrén B. Randomized clinical trial of the effect of interferon alpha on survival in patients with disseminated midgut carcinoid tumours. Br J Surg. 2003;90(6):687-693.

16. Faiss S, Scherübl H, Riecken EO, Wiedenmann B. Interferon-alpha versus somatostatin or the combination of both in metastatic neuroendocrine gut and pancreatic tumours. Digestion. 1996;57 Suppl 1:84-85.

17. Arnold R, Rinke A, Klose KJ, et al. Octreotide versus octreotide plus interferon-alpha in endocrine gastroenteropancreatic tumors: a randomized trial. Clin Gastroenterol Hepatol. 2005;3(8):761-771.

18. Modlin IM, Pavel M, Kidd M, Gustafsson BI. Review article: somatostatin analogues in the treatment of gastroenteropancreatic neuroendocrine (carcinoid) tumours. Ailment Pharmacol Ther. 2010;31(2):169-188.

19. Grozinsky-Glasberg S, Shimon I, Korbonits M, Grossman AB. Somatostatin analogues in the control of neuroendocrine tumours: efficacy and mechanisms. Endocr Relat Cancer. 2008;15(3):701-720.
20. Rinke A, Müller HH, Schade-Brittinger C, et al; PROMID Study Group Placebo-controlled, double-blind, prospective, randomized study on the effect of octreotide LAR in the control of tumor growth in patients with metastatic neuroendocrine midgut tumors: a report from the PROMID Study Group. J Clin Oncol. 2009;27(28):4656-4663.

21. Arnold R, Wittenberg M, Schade-Brittinger C, et al; PROMID Study Group. Placebo controlled, double blind, prospective, randomized study on the effect of octreotide LAR in the control of tumor growth in patients with metastatic neuroendocrine midgut tumors (PROMID): results on long-term survival [abstract]. J Clin Oncol. 2013;31 Suppl:abstract 4030 .

22. Lepage C, Bouvier AM, Manfredi S, Dancourt V, Faivre J. Incidence and management of primary malignant small bowel cancers: a well-defined French population study. Am J Gastroenterol. 2006; 101(12):2826-2832.

23. Ploeckinger U, Kloeppel G, Wiedenmann B, Lohmann R; representatives of 21 German NET Centers. The German NET-registry: an audit on the diagnosis and therapy of neuroendocrine tumors. Neuroendocrinology. 2009;90(4):349-363.

24. Caplin ME, Pavel M, Ćwikla JB, eta 1; CLARINET Investigators. Lanreotide in metastatic enteropancreatic neuroendocrine tumors. $N$ Engl J Med. 2014;371(3):224-233.
OncoTargets and Therapy

\section{Publish your work in this journal}

OncoTargets and Therapy is an international, peer-reviewed, open access journal focusing on the pathological basis of all cancers, potential targets for therapy and treatment protocols employed to improve the management of cancer patients. The journal also focuses on the impact of management programs and new therapeutic agents and protocols on

\section{Dovepress}

patient perspectives such as quality of life, adherence and satisfaction. The manuscript management system is completely online and includes a very quick and fair peer-review system, which is all easy to use. Visit http://www.dovepress.com/testimonials.php to read real quotes from published authors. 